\title{
Isobutyl Isothiocyanate is a Potent Heat Tolerance Enhancer for Arabidopsis
}

\author{
Masakazu Hara, Takumi Matsubara, Ikuo TAkahashi and Hiroki Murano \\ Research Institute of Green Science and Technology, Shizuoka University, 836 Ohya, Shizuoka 422-8529, Japan
}

(Received April 19, 2017; Accepted January 22, 2018)

\begin{abstract}
Heat tolerance enhancers are chemicals which prevent the physiological damages of plants by heat. Phenethyl isothiocyanate (ITC), which is known as the heat tolerance enhancer, induced the expression of heat shock protein genes in Arabidopsis. Here we measured the heat shock response (HSR)-inducing activities of ITCs to find more active ITCs than phenethyl ITC. We prepared transgenic Arabidopsis possessing the $\beta$-glucuronidase reporter gene driven by the promoter of a small heat shock protein (HSP17.6C-CI) gene which was induced by heat tolerance enhancers. We tested 16 naturally occurring ITCs for their HSR-inducing activities: 5 aromatic ITCs, 7 aliphatic ITCs, and 4 sulfur-containing aliphatic ITCs. The results showed that the short-chain aliphatic ITCs, especially isobutyl ITC, promoted the HSR more strongly than the aromatic and sulfur-containing aliphatic ITCs. Isobutyl ITC induced the HSR in the whole Arabidopsis seedlings. The isobutyl ITC-treated plants showed higher heat tolerance than the phenethyl ITC-treated plants. These results suggest that isobutyl ITC is a more potent heat tolerance enhancer than phenethyl ITC.
\end{abstract}

Keywords : heat shock protein, heat shock response, heat tolerance enhancers

\section{INTRODUCTION}

Exposure to extreme and prolonged heat is one of the major causes of damage to crop production. Heat stress allows plants to exacerbate various symptoms such as protein denaturation and degradation, increased fluidity of membrane lipids, the production of reactive oxygen species, and the suppression of photosynthesis (Wahid et al., 2007). Plants ameliorate the stress symptoms by physiological countermeasures, including production of compatible solutes, activation of antioxidative systems, and expression of heat shock proteins (HSPs) (Bita and Gerats, 2013). Among these countermeasures, HSPs are major factors in enhancing heat tolerance in plants, because the expression levels of HSP genes in plants are positively correlated with the degree of heat tolerance (Wahid et al., 2007; Waters, 2013).

Although heat is a potent trigger for the heat shock response (HSR) in organisms, other stresses (i.e., osmotic stress, oxidative stress, irradiations, wounding, and chemicals) also induce HSR (Sørensen et al., 2003; Al-Whaibi, 2011). Natural compounds have been reported to exert inducing effects on the HSR in plants. It has been demonstrated that the application of salicylic acid (SA) (Dat et al., 1998), geldanamycin (GDA) (Yamada et al., 2007), sanguinarine (Hara and Kurita, 2014), and (E)-2-hexenal and its related compounds (Yamauchi et al., 2015) have been shown to induce the expression of plant HSP genes and enhance the heat tolerance of plants. In Arabidopsis, the HSP17.6C-CI (AtIg53540) gene which belongs to the small HSP family was highly upregulated by sanguinarine (Hara and Kurita, 2014) and (E)-2-hexenal (Yamauchi et al., 2015), suggesting that this gene is a common regulon controlled by chemical HSR inducers in Arabidopsis.

Isothiocyanates (ITCs) are sulfur-containing metabolites mainly formed from Brassicaceae plants. They are produced through the hydrolysis of the corresponding precursors, i.e., glucosinolates by myrosinase. Biochemical and genetic studies have demonstrated the reaction mechanism of the glucosinolate-myrosinase system, the tissue and cellular localization of myrosinase and glucosinolate, and the biosynthetic pathways of glucosinolates (Halkier and Gershenzon, 2006; Kissen et al., 2009). It has been considered that the production of ITCs is a part of the defense mechanisms of plants, because ITCs show anti-microorganism, animal-repellent, allelopathic, and herbicidal activities (Halkier and Gershenzon, 2006; Hopkins et al., 2009; Hara et al., 2010). Recently, we found that phenethyl ITC enhanced heat tolerance of Arabidopsis (Hara et al., 2013). Since ITCs are contained in food condiments, ITCs are promising as heat tolerance enhancers. However, little is known which ITCs show more HSR-inducing activities than phenethyl ITC.

In this study, we tested 16 kinds of ITCs including phenethyl ITC for their HSR-inducing activities by using the transgenic Arabidopsis which has the $\beta$-glucuronidase (GUS) reporter gene controlled by the HSP17.6C-CI gene promoter. We found that the ITCs with short branched side chains showed more potent activation of the HSP 17.6C-CI gene promoter rather than phenethyl ITC.

\footnotetext{
Corresponding author : Masakazu Hara, fax: +81-54-238-5134, e-mail : hara.masakazu@shizuoka.ac.jp
}

"Present address: Graduate School of Agricultural and Life Sciences, The University of Tokyo, Tokyo, Japan 


\section{MATERIALS AND METHODS}

\section{Chemicals}

Phenethyl ITC (1), benzyl ITC (3), 4-methoxybenzyl ITC (5), hexyl ITC (11), methallyl ITC (12), ethyl ITC (7), butyl ITC (8), isopropyl ITC (9), and 3-(methylthio) propyl ITC (14) were obtained from Sigma-Aldrich (Tokyo, Japan). Phenyl ITC (2), allyl ITC (6), and isobutyl ITC (10) were purchased from WAKO (Tokyo, Japan). 4-Phenylbutyl ITC (4), sulforaphane (13), alyssin (15), and sulforaphene (16) were from LKT Labs (St. Paul, MN, USA). 4Methylumbelliferyl- $\beta$-D-glucuronide (4-MUG), 4-methylumbelliferone (4-MU), and 5-bromo-4-chloro-3-indolyl$\beta$-D-glucuronide (X-Gluc) were obtained from WAKO. GDA was purchased from TokyoKasei (Tokyo, Japan).

\section{Production of transgenic Arabidopsis}

A plasmid vector for the transformation of Arabidopsis thaliana was constructed by exchanging the cauliflower mosaic virus (CaMV) 35S promoter region, including AtADH 5'-UTR of pRI201-AN-GUS (Takara, Shiga, Japan), to the $1-\mathrm{kb}$ upstream region from the translational start site of the HSP17.6C-CI (Atlg53540) gene. The pRI201-AN-GUS plasmid was digested by HindIII and NdeI to remove the CaMV 35S promoter and AtADH 5'UTR regions. The upstream region of the HSP17.6C-CI gene, which was amplified by polymerase chain reaction, was inserted via the sticky ends of HindIII and NdeI to the plasmid using the In-Fusion kit (Takara) according to the manufacturer's instruction. Finally, the HSP17.6C-CI gene promoter was located adjacent to the 5 '-terminus of the GUS gene of the pRI201-AN-GUS plasmid. We designated the plasmid HSP17.6C-CIProGUS. Arabidopsis thaliana (L.) Heynh. ecotype Columbia (Col-0) was transformed with the HSP17.6C-CIProGUS plasmid using the Agrobacterium-mediated floral dip method. The homozygotes were obtained and named the HSP17.6C-CIProGUS Arabidopsis plant.

GUS reporter assay

The GUS activity assay was performed according to the established method of whole-plant GUS reaction (Weigel and Glazebrook, 2002), with slight modifications. Seeds of the HSP17.6C-CIProGUS Arabidopsis were sown on a $1 / 5$ MS medium containing $1 \%$ sucrose solidified by $0.8 \%$ agar in 6 -well plates (TPP, Trasadingen, Switzerland, \#92406) under sterile conditions. The plates were kept at $6{ }^{\circ} \mathrm{C}$ for $2 \mathrm{~d}$, and then transferred to the growth chamber (NK System, Tokyo, Japan), conditioned at $22^{\circ} \mathrm{C}$ with a 16-h day $\left(60 \mu \mathrm{mol} \mathrm{m} \mathrm{m}^{-2} \mathrm{~s}^{-1}\right) / 8$-h night cycle. At $6 \mathrm{~d}$ after germination (DAG), whole seedlings were soaked in $250 \mu \mathrm{L}$ of test solution in $1.5-\mathrm{mL}$ micro test tubes ( 2 seedlings per tube). The test solutions were $1 \%$ dimethyl sulfoxide $(\mathrm{DMSO})$ in water $(\mathrm{v} / \mathrm{v})$ containing $\operatorname{GDA}(5,50$, and $500 \mu \mathrm{M})$ and ITCs $(0.02,0.1,0.2,1$, and $2 \mathrm{mM})$. Test tubes were incubated at $22^{\circ} \mathrm{C}$ for $6 \mathrm{~h}$ under illumination $\left(60 \mu \mathrm{mol} \mathrm{m} \mathrm{m}^{-2} \mathrm{~s}^{-1}\right)$. Control solution was $1 \%$ DMSO. For heat shock (HS), the 6 DAG seedlings soaked in the $1 \%$ DMSO solution of the test tubes were incubated at $22^{\circ} \mathrm{C}$ for $5 \mathrm{~h}$, and then the test tubes were immersed in a water bath at $37^{\circ} \mathrm{C}$ for $1 \mathrm{~h}$. The seedlings that were rinsed with water were transferred to the wells ( 2 seedlings per well) in a 96-well microplate (IWAKI, Funabasi, Japan) containing $200 \mu \mathrm{L}$ of the GUS assay solution composed of $50-\mathrm{mM}$ sodium phosphate buffer $\mathrm{pH} 7.0,10-\mathrm{mM}$ EDTA, 0.1\% (w/v) Triton X-100, 0.1\% (w/v) SDS, and 1-mM 4-MUG. Seedlings were incubated at $22^{\circ} \mathrm{C}$ for $0,1,2,4,16,24$, and $48 \mathrm{~h}$ in the dark. At the end of incubations, $100 \mu \mathrm{L}$ of $1 \mathrm{M}$ $\mathrm{Na}_{2} \mathrm{CO}_{3}$ was added and incubated for $10 \mathrm{~min}$ to stop the GUS reaction and enhance the fluorescence of the reaction product (4-MU). The solutions were appropriately diluted with $0.33-\mathrm{M} \mathrm{Na}_{2} \mathrm{CO}_{3}$. The fluorescence was determined at an excitation and emission wavelength of 365 and $455 \mathrm{~nm}$, respectively, with Varioskan Flash (Thermo Fisher Scientific, Yokohama, Japan). The 4-MU amounts were calculated from the calibration curve made with authentic 4MU. When ITCs were tested, the incubation period in the GUS assay solution was $16 \mathrm{~h}$.

GUS staining of Arabidopsis seedlings was done as previously described (Weigel and Glazebrook, 2002). The 7 DAG seedlings were treated with isobutyl ITC $(0.2 \mathrm{mM})$ as described above for $6 \mathrm{~h}$. The control was without the ITC. After destaining with acetone, the seedlings were stained with X-Gluc, and then observed with light microscopy.

Heat-tolerance experiment

Seeds of the wild Arabidopsis were sown on filter paper (No. 1, Advantec, Tokyo, Japan), which was then plated on the $1 / 5 \mathrm{MS}$ medium containing $1 \%$ sucrose solidified by $0.8 \%$ agar in 6-well plates (TPP, \#92406) under sterile conditions. The plates were kept at $6^{\circ} \mathrm{C}$ for $2 \mathrm{~d}$ (vernalization), and then transferred to the growth chamber (NK System, Japan) conditioned at $22^{\circ} \mathrm{C}$ with a $16-\mathrm{h} \mathrm{d}(60$ $\left.\mu \mathrm{mol} \mathrm{m} \mathrm{m}^{-2} \mathrm{~s}^{-1}\right) / 8$-h night cycle. At 6 DAG, the 6 seedlings with the filter paper were inoculated onto the test medium in a $60-\mathrm{mm}$ plastic Petri dish (IWAKI, Japan).

Test ITCs were prepared as DMSO solution at concentrations of $0.25,2.5,25$, and $250 \mathrm{mM}$. To make the test medium ( $8 \mathrm{ml}$ in each Petri dish), the test ITC DMSO solutions $(16 \mu \mathrm{l})$ were added to the $1 / 5 \mathrm{MS}$ medium with $0.8 \%$ agar and $1 \%$ sucrose $(7984 \mu \mathrm{l})$. The final concentrations of the test medium were $0.5,5,50$, and $500 \mu \mathrm{M}$, respectively. The Petri dishes were sealed with Parafilm to prevent the emission of ITCs from the dish. After incubating the seedlings with the test medium under the same conditions as above for $24 \mathrm{~h}$, the plates were sealed with a polyethylene zipper bag (4-F, $120 \mathrm{~mm} \times 170 \mathrm{~mm}$, System Polymer, Tokyo, Japan) and soaked in water at $46^{\circ} \mathrm{C}$ for 1 h. Control plates were placed in the same growth chamber for $1 \mathrm{~h}$. The dishes containing heat-treated and control plants were maintained for $3 \mathrm{~d}$ under the described conditions. At $10 \mathrm{DAG}$, the seedlings were harvested, and the fresh weights were measured. Chlorophyll content was determined spectrophotometrically according to the common method (Wintermans and de Mots, 1965).

Statistical analysis

Data for $P$ values were analyzed by Student's $t$-test at a significance level of 0.05 . 


\section{RESULTS AND DISCUSSION}

Reporter gene assay

First, we produced transgenic Arabidopsis plants to conduct the reporter assay for HSR in plants. The construct of the transgene is shown in Fig. 1a. We used the promoter of a small HSP gene, i.e., the HSP17.6C-CI (At1g53540) gene of Arabidopsis. A previous study reported that the HSP17.6C-CI gene was upregulated by the addition of GDA (Hara and Kurita, 2014). The upstream region (1 kb) of the HSP17.6C-CI gene was inserted into the site adjacent to the $\beta$-glucuronidase (GUS) reporter gene, which was followed by the heat shock protein terminator. The transgenic Arabidopsis was designated as the HSP17.6CCIProGUS plant.

Induction of the HSR by the GDA treatment in the HSP17.6C-CIProGUS plant is shown in Fig. 1b. After treating the HSP17.6C-CIProGUS plant with GDA for $6 \mathrm{~h}$, the plants were inoculated from the GDA solution to the GUS reaction solution containing 4-MUG, which can be converted to fluorescent 4-MU by GUS. It has been established that GUS activity can be detected without extracting the GUS protein from the plants, because both 4-MUG and 4-MU can pass through the plant cell membranes under the reported conditions (Weigel and Glazebrook, 2002). The GDA addition gradually enhanced 4-MU production during the incubation period. GDA concentration of $50 \mu \mathrm{M}$ promoted 4-MU production more than concentrations of 5 and $500 \mu \mathrm{M}$. The blank control (0 $\mu \mathrm{M}$ GDA) enhanced 4MU production very little. Heat $\left(37^{\circ} \mathrm{C}\right.$ for $\left.1 \mathrm{~h}\right)$ induced more 4-MU production in the HSP17.6C-CIProGUS plant than the GDA treatment (Fig. 1c). Because the HSP17.6C-
CI promoter responded to both the GDA addition and heat, this reporter assay was available to analyze the activity of HSR induction by ITCs.

Induction of HSR in Arabidopsis by ITCs

We measured the HSR-inducing activities of the naturally occurring ITCs listed in Fig. 2 using the reporter assay. We tested 16 ITCs, including 5 aromatic, 7 aliphatic, and 4 sulfur-containing aliphatic ITCs. After the HSP17.6C-CIProGUS plants were treated with the ITCs for $6 \mathrm{~h}, 4-\mathrm{MU}$ productions by GUS were determined at the 16th $\mathrm{h}$ of incubation in the whole-plant reaction. Although all 16 ITCs promoted 4-MU production, promoting effect varied by type of ITC (Fig. 3). Overall, the aliphatic ITCs tended to enhance 4-MU production more strongly than the aromatic and sulfur-containing aliphatic ITCs. In particular, isobutyl ITC (10) and isopropyl ITC (9) potently promoted 4-MU production. The following tests were done using isobutyl ITC (10), because the highest 4-MU production was marked by the administration of isobutyl ITC (10) at $0.2 \mathrm{mM}$ in Fig. 3. GUS staining was clearly observed in almost the whole HSP17.6C-CIProGUS plant treated with isobutyl ITC (10) (Fig. 4), suggesting that most tissues of Arabidopsis seedlings could exhibit the HSR to the application of isobutyl ITC (10).

Enhancement of heat tolerance by ITCS

One day after the addition of ITCs to the wild-type Arabidopsis seedlings (6 DAG), the plants were treated with heat stress $\left(46^{\circ} \mathrm{C}\right.$ for $\left.1 \mathrm{~h}\right)$. The heat-stressed seedlings were cultivated for $3 \mathrm{~d}$, and then fresh weight and chlorophyll content were measured (Fig. 5a). Both the fresh weight and chlorophyll content of the seedlings treated with $500-\mu \mathrm{M}$ isobutyl ITC (10) were significantly higher than those of the control $(0 \mu \mathrm{M})$ seedlings (Fig. $5 \mathrm{~b}, \mathrm{c})$.

(a)
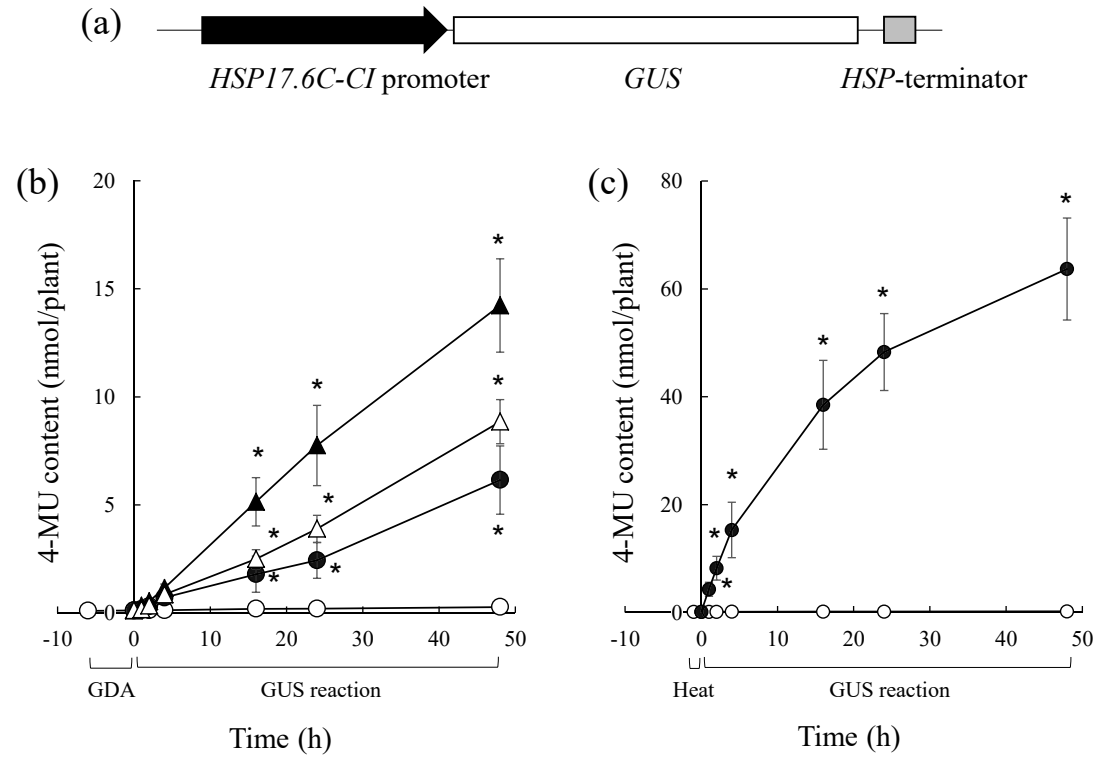

Fig. 1 GUS reporter assay. (a) Construct of the transgene in the HSP17.6C-CIProGUS plasmid. (b) Time course of the 4-MU production by the HSP17.6C-CIProGUS Arabidopsis plant treated with GDA. The concentrations were $0 \mu \mathrm{M}$ (open circles), $5 \mu \mathrm{M}$ (closed circles), $50 \mu \mathrm{M}$ (closed triangles), and $500 \mu \mathrm{M}$ (open triangles), respectively. (c) Heat shock $\left(37^{\circ} \mathrm{C}\right.$ for $1 \mathrm{~h}$ ) was applied to the HSP17.6C-CIProGUS plants. Closed and open circles show heat shock and control, respectively. Values and bars represent means $\pm \mathrm{SD}$ (four individual experiments). Asterisks show significant differences $(P<0.05)$ as determined by Student's $t$-test in a comparison between no treatment $(0 \mu \mathrm{M})$ and treatments. 


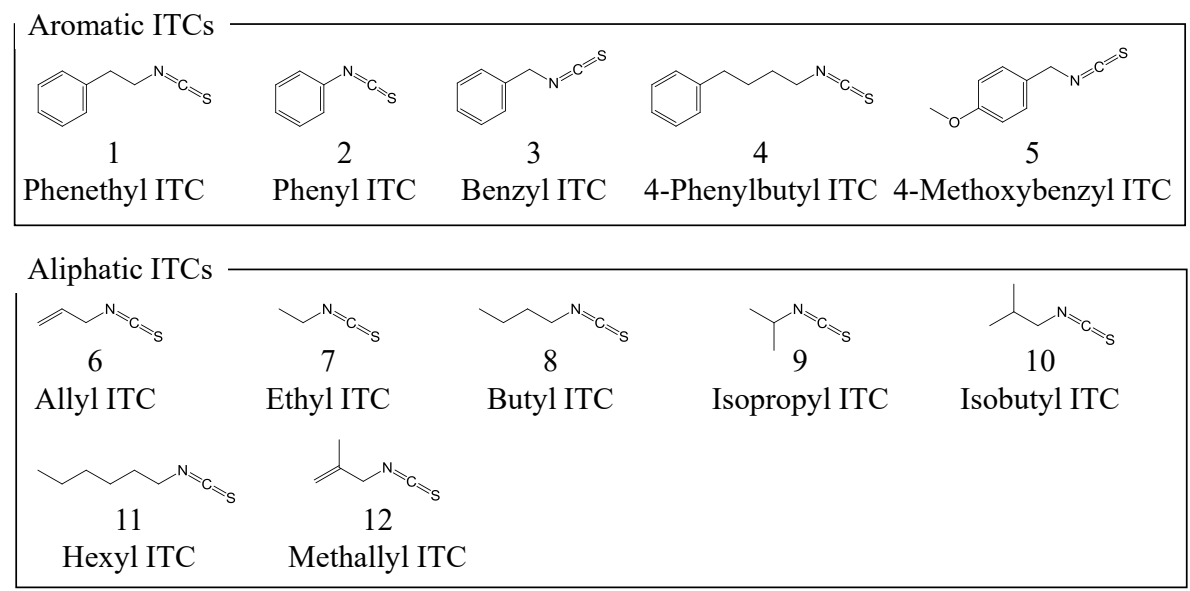

Sulfur-containing aliphatic ITCs

\begin{tabular}{|c|c|c|c|}
\hline 13 & 14 & 15 & 16 \\
\hline Sulforaphane & 3-(Methylthio) propyl ITC & Alyssin & Sulforaphene \\
\hline
\end{tabular}

Fig. 2 Sixteen ITCs used in this study.

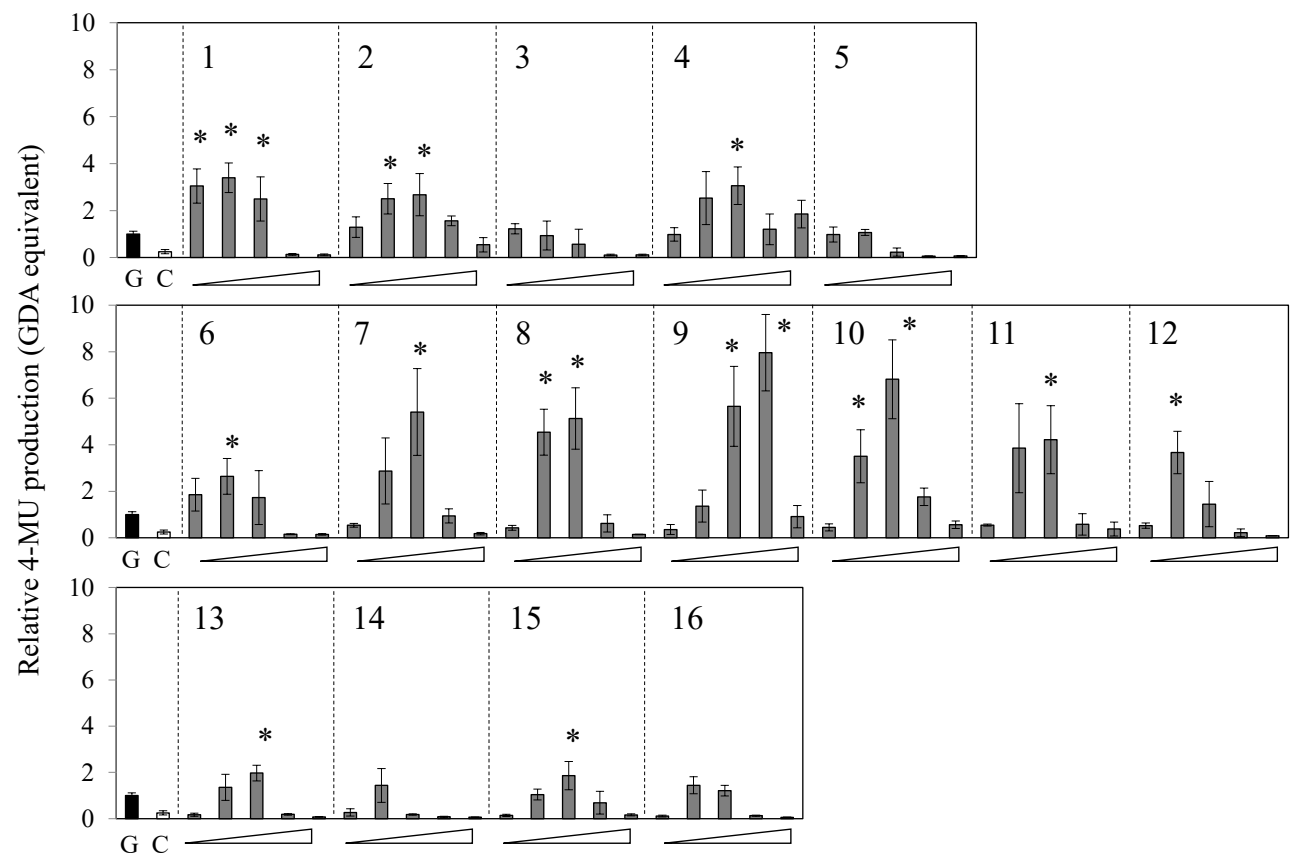

Fig. 3 Promotion of HSR by ITCs in Arabidopsis. Numbers represent ITCs corresponding to the numbers in Fig. 2. The 4-MU productions were determined after whole-plant GUS reaction for $16 \mathrm{~h}$. Values are shown as GDA equivalents standardized to the values obtained by the GDA treatment. ITCs were applied at concentrations of $0.02,0.1,0.2,1$, and $2 \mathrm{mM}$, from left to right, respectively, as shown by open triangles. Black and white bars show GDA treatment $(\mathrm{G}, 0.05 \mathrm{mM})$ and control $(\mathrm{C}, 0 \mathrm{mM}$ ITC), respectively. Values and bars represent means \pm SD (three individual experiments). Asterisks show significant high values $(P<0.05)$ as determined by Student's $t$-test in a comparison between GDA and ITC treatments.

However, such effects were not observed when phenethyl ITC (1) was used. The heat condition $\left(46^{\circ} \mathrm{C}\right.$ for $\left.1 \mathrm{~h}\right)$ was lethal for the phenethyl ITC-treated seedlings. This indicates that isobutyl ITC showed the higher heat tolerance-enhancing effect than phenethyl ITC in the experimental condition.

Use of ITCs with short-branched side chains

The reporter assay indicated that aliphatic ITCs showed stronger HSR-inducing activities than aromatic and sulfur-containing aliphatic ITCs, whereas most ITCs had more activities than GDA. Since most aliphatic ITCs have smaller molecular weights than aromatic and sulfurcontaining aliphatic ITCs, aliphatic ITCs may more easily access the active sites of the plant cell. Among aliphatic ITCs, allyl ITC (6) and methallyl ITC (12) showed weaker activities than other aliphatic ITCs, suggesting that aliphatic ITCs possessing carbon-carbon double bonds may be chemically unstable compared to aliphatic ITCs without 


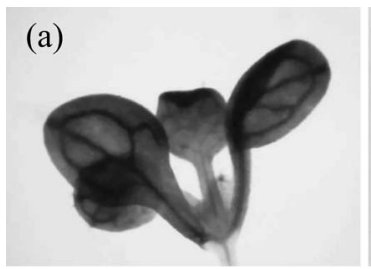

(d)
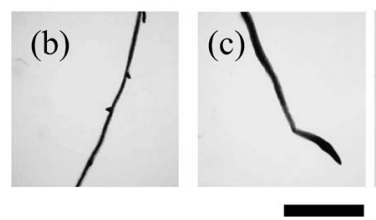

(e)

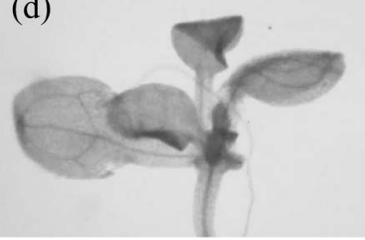

(f)

Fig. 4 GUS staining of Arabidopsis seedlings. Seedlings were treated with $0.2-\mathrm{mM}$ isobutyl ITC (a, b, and c) and without ITC (d, e, and f). Shoots (a and d), main roots (b and e), and the tips of the main roots (c and f) are shown. The scale bars are $5 \mathrm{~mm}$.

the double bond.

Since ITCs are potent electrophiles which can react with thiol groups of various proteins and glutathione, ITCs may affect protein homeostasis and redox balance in the cell (Murakami, 2013). These effects may be triggers for inducing the HSR. Besides that, it was recently reported that sulforaphane bound to HSP90 and disrupted the HSP90-p50 ${ }^{\text {Cdc37 }}$ complex in pancreatic cancer cells ( $\mathrm{Li}$ et al., 2012). In Arabidopsis, HSP90 is thought to suppress HsfA1, which is a transcription factor for many HSP genes, including HSP17.6C-CI (Yoshida et al., 2011), by binding it. It is postulated that when the action of HSP90 was inhibited by HSP90 inhibitors such as GDA, HsfA1 could be released and activated by disrupting the interaction between HSP90 and HsfA1 (Yamada et al., 2007). It is supposed that ITCs may induce the HSP17.6C-CI gene expression by activating HsfA1 via disrupting the HSP90HsfA1 interaction.

ITCs with short-branched side chains are found in many plant species (Fahey et al., 2001). Isobutyl ITC (10) is a pungent component of horseradish. Isopropyl ITC (9) is contained in Brassicaceae vegetables. This means that these ITCs are safe because they have been taken by humans for a long period. ITCs with short-branched side chains may be promising for the practical enhancers of heat tolerance in plants.

\section{ACKNOWLEDGEMENTS}

This work was partially supported by A-STEP, Japan Science and Technology Agency (JST).

\section{REFERENCES}

Al-Whaibi, M. H. 2011. Plant heat-shock proteins: A mini review. J. King. Saud. Univ. Sci. 23: 139-150.

Bita, C. E., Gerats, T. 2013. Plant tolerance to high temperature in a changing environment: scientific fundamentals and production of heat stress-tolerant crops. Front. Plant Sci. 4: 273.

Dat, J. F., Lopez-Delgado, H., Foyer, C. H., Scott, I. M. 1998. Parallel changes in $\mathrm{H}_{2} \mathrm{O}_{2}$ and catalase during thermotolerance induced by salicylic acid or heat acclimation in mustard seedlings. Plant Physiol. 116: 1351-1357.

Fahey, J. W., Zalcmann, A. T., Talalay, P. 2001. The chemical diversity and distribution of glucosinolates and isothiocyanates among plants. Phytochemistry 56: 5-51.

Halkier, B. A., Gershenzon, J. 2006. Biology and biochemistry of glucosinolates. Annu. Rev. Plant Biol. 57:303-333.

Hara, M., Yatsuzuka, Y., Tabata, K., Kuboi, T. 2010. Exogenously applied isothiocyanates enhance glutathione $S$-transferase expression in Arabidopsis but act as herbicides at higher

(a)

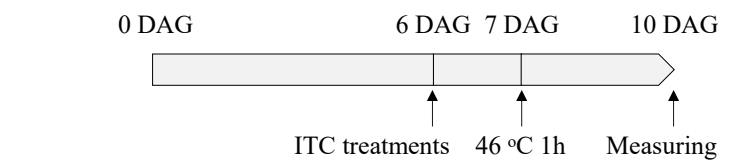

(b)

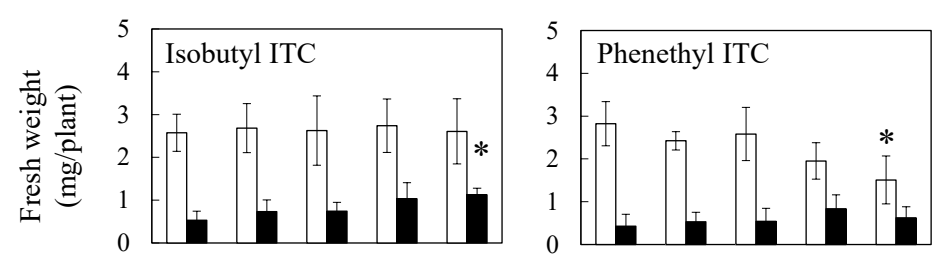

(c)

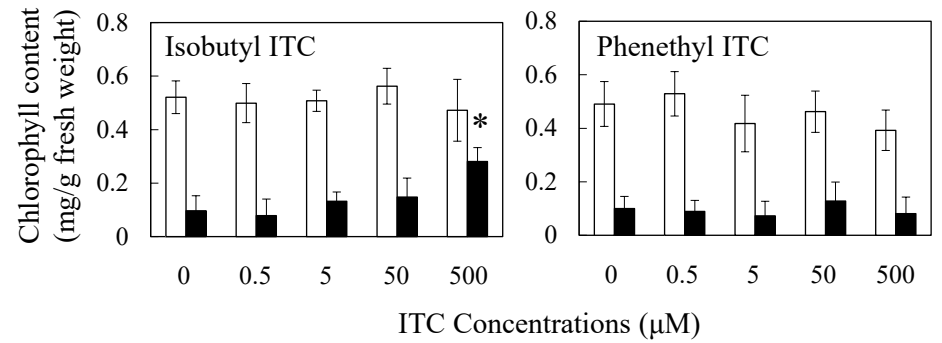

Fig. 5 Effects of pre-treatments with isobutyl ITC and phenethyl ITC on fresh weights and chlorophyll contents of Arabidopsis seedlings after heat $\left(46^{\circ} \mathrm{C}\right.$ for $1 \mathrm{~h}$ ). (a) The scheme of the experimental schedule. DAG means days after germination. The fresh weight (b) and the chlorophyll content (c) of seedlings. White and black bars represent plants not exposed to heat and plants exposed to heat, respectively. Values and bars represent means $\pm \mathrm{SD}(n=12$ in fresh weight and $n=4$ in chlorophyll content). Asterisks show significant differences $(P<0.05)$ as determined by Student's $t$-test in a comparison between no treatment $(0 \mu \mathrm{M})$ and the ITC treatments. 
concentrations. J. Plant Physiol. 167: 643-649.

Hara, M., Harazaki, A., Tabata, K. 2013. Administration of isothiocyanates enhances heat tolerance in Arabidopsis thaliana. Plant Growth Regul. 69: 71-77.

Hara, M., Kurita, I. 2014. The natural alkaloid sanguinarine promotes the expression of heat shock protein genes in Arabidopsis. Acta Physiol. Plant. 36: 3337-3343.

Hopkins, R. J., van Dam, N. M., van Loon, J. J. 2009. Role of glucosinolates in insect-plant relationships and multitrophic interactions. Annu. Rev. Entomol. 54: 57-83.

Kissen, R., Rossiter, J. T., Bones, A. M. 2009. The 'mustard oil bomb' : not so easy to assemble?! Localization, expression and distribution of the components of the myrosinase enzyme system. Phytochem. Rev. 8: 69-86.

Li, Y., Karagoz, G. E., Seo, Y. H., Zhang, T., Jiang, Y., Yu, Y., Duarte, A. M., Schwartz, S. J., Boelens, R., Carroll, K., Rüdiger, S. G., Sun, D. 2012. Sulforaphane inhibits pancreatic cancer through disrupting Hsp90-p50(Cdc37) complex and direct interactions with amino acids residues of Hsp90. J. Nutr. Biochem. 23: 1617-1626.

Murakami, A. 2013. Modulation of protein quality control systems by food phytochemicals. J. Clin. Biochem. Nutr. 52: 215-227.

Sørensen, J. G., Kristensen, T. N., Loeschcke, V. 2003. The evolutionary and ecological role of heat shock proteins. Ecol. Lett. 6: $1025-1037$.
Wahid, A., Gelani, S., Ashraf, M., Foolad, M. R. 2007. Heat tolerance in plants: An overview. Environ. Exp. Bot. 61: 199-223.

Waters, E. R. 2013. The evolution, function, structure, and expression of the plant sHSPs. J. Exp. Bot. 64: 391-403.

Weigel, D., Glazebrook, J. 2002. Arabidopsis: A laboratory manual. Cold Spring Harbor Laboratory Press, New York, pp 251-252.

Wintermans, J. F., de Mots, A. 1965. Spectrophotometric characteristics of chlorophylls a and $\mathrm{b}$ and their pheophytins in ethanol. Biochim. Biophys. Acta 109: 448-453.

Yamada, K., Fukao, Y., Hayashi, M., Fukazawa, M., Suzuki, I., Nishimura, M. 2007. Cytosolic HSP90 regulates the heat shock response that is responsible for heat acclimation in Arabidopsis thaliana. J. Biol. Chem. 282:37794-37804.

Yamauchi, Y., Kunishima, M., Mizutani, M., Sugimoto, Y. 2015. Reactive short-chain leaf volatiles act as powerful inducers of abiotic stress-related gene expression. Sci. Rep. 26: 8030.

Yoshida, T., Ohama, N., Nakajima, J., Kidokoro, S., Mizoi, J., Nakashima, K., Maruyama, K., Kim, J. M., Seki, M., Todaka, D., Osakabe, Y., Sakuma, Y., Schöffl, F., Shinozaki, K., Yamaguchi-Shinozaki, K. 2011. Arabidopsis HsfA1 transcription factors function as the main positive regulators in heat shock-responsive gene expression. Mol. Genet. Genomics 286: $321-332$. 